more specifically technical subjects that their full cultural possibilities may be realized. The curriculum, moreover, must be widened. There has been a rapid movement in this direction during the past thirty years, a movement to which evacuation experiences have given an added drive; many schoolmasters, evacuated to a new environment and compelled by the exigencies of war to devote the time of their pupils to activities which have never hitherto fallen within their purview, have found, to their surprise, that these activities are often more educational than the class-room subjects whose time they have usurped. It is to be hoped that this lesson of evacuation will never be forgotten. But a wider curriculum can only be maintained if it is accompanied by greater freedom of choice on the part of the boys and girls for whom it is provided. We need a much greater variety in post-primary education, whether this takes the form of a greater variety of schools or a greater variety of 'sides' within the same school. I can visualize a postprimary education in the future of which the staple constituent will be book-learning as in the secondary school with which we are familiar, and at the other end of the scale an education in which book-learning will be almost non-existent, with infinite gradations in between: I visualize schools which are more like clubs, new types of boarding-schools (on the lines of the present camp-schools), schools which are day schools up to a certain age and boarding-schools beyond that, and many other varieties. Only in such a variegated field shall we be able to attain that 'equality of opportunity' in education which is to-day a popular slogan, but like most slogans very illthought out. It does not mean equality of opportunity to attend a certain type of school enjoying a fictitious prestige, or equality of opportunity to go to the university. It means that every child should enjoy an equal opportunity with every other child to receive the right education for him, and that means that we must have available types of education to meet the needs, intellectual, moral, physical, and spiritual, of all children.

My third and final principle is that, in a far more real sense than ever before, the schools should serve the community, and the community the schools. The community demands that the product of education should be the good citizen; the individual demands the development of a unique personality. These demands are often regarded as contradictory; they are in fact complementary, for the fully developed individual is the life-blood of democracy, and the best citizen is the man who has been given the opportunity, and has taken it, to become his best self. This truth is more likely to be appreciated, and the duty of the community to the school and of the school to the community is more likely to be performed, if there is a much closer association between the two. On one hand there should be no schools outside the national system. This does not mean standardization, or the reduction of all schools to the dead level of the few categories which we know; for the national system itself is capable of expansion to embrace a much wider variety of schools than it does to-day, and there should be room within it for all those diversities of educational life and experiment represented by schools which are at present independent of public support: given public support, they must, of course, be open to all who can profit by the education they offer, and our ideal should be schools common to all classes but diversified in type.

The two essential freedoms to safeguard are the freedom of the parent to choose his child's school, and the freedom of the school to choose its pupils, both freedoms being exercised within wide limitations. This association between school and com. munity can be further fostered by the encouragement within the school itself of realistic social studies. I do not mean by this courses in civics or current affairs, though these may have their importance. But citizenship is best learnt by practising it within the school community, and its significance can best be appreciated by the study and use of the community immediately outside the school walls. The locality should, in fact, be the first text-book of every school. If that can be done, there will be less danger of that community-sense which many of our schools foster (and particularly our boarding-schools) stopping short, as it so often does, at a school-communitysense, and a bridge will be built (one of the most difficult bridges to build) between school and 'real life'. 'That should be the beginning of an outward movement which will end in the world-community claiming the allegiance of the world-citizen.

Finally, the 'walls of partition' must be broken down from the outside as well as from the inside, and if the school is to go out to the community, the community must come into the school. To some extent this is being done, and it is symbolical of the change that, whereas in the nineteenth century schools were surrounded by high walls and locked gates, to-day they are commonly built open to the streets and the gates are rarely shut. But the flow of traffic through this open gate is not yet as free as it should be. There is no reason why the industry and the craftsmanship of the neighbourhood should not find their way into the classroom and display their wares and expound their ways of life there-invaluable recruits to the teaching profession; there is no reason why other recruits should not be found among representatives of the local authority and of the professions; there is no reason why the schools themselves should not become community-centres for education, providing classes and courses and cultural activities for children in the day-time and for their parents in the evenings. In these ways social studies would become a real and vital element in the curriculum, and the association between school and community would be complete.

Much has necessarily been left unsaid in this article. No mention has been made of the universities or of adult education : the whole field of religious education has been left unexplored-if explored, it would be found to affect profoundly not only the curriculum, but also the whole organization and administration of the school ; and nothing has been said about the training of teachers or the relationship between teaching and other forms of youth service. But I hope I have succeeded in erecting certain pointers to the education of to-morrow.

\section{STRUCTURE AND ROTATION OF GALAXIES}

A

SUCCESSFUL and well-attended symposium on the structure and rotation of our own Milky Way system and of external galaxies was held at the rooms of the Royal Astronomical Society in Burlington House on July 10.

The discussion opened with a contribution by Dr. A. Hunter on interstellar absorption and the scale of 
the Galaxy. Pointing out that the absorption produced by interstellar matter is important in studies of galactic structure because of the effect which it may have on estimates of stellar distance, Dr. Hunter went on to review modern methods of determining great distances in astronomy. Except within about a hundred light-years of the sun, the distance of a star is found by comparing its apparent brightness with its true brightness, this latter being estimated by physical examination of the star's light. The distance then follows by applying the inverse-square law. If this law does not hold, however-if, for example, we attribute wholly to the effect of distance dimming which is partly due to absorption-we shall over-estimate distances by amounts which may be very serious if the absorption is heavy. In the past decade many attempts have been made to determine the absorption in various directions. Nearly all depend on comparing photometric distances of distant objects determined as outlined above with distances inferred by geometrical means. Trumpler, for example, arguing that the diameters of physically similar clusters ought to appear smaller in proportion to their distance, found that distances so estimated could only be reconciled with photometric distances by assuming an average absorption of 0.67 stellar magnitudes for every thousand parsecs.

Other work on broadly similar lines has more recently led to much the same result for observations made close to the galactic equator. Hubble's results on the dimming of extra-galactic nebulæ by absorption in our own system suggest that the obscuring matter is confined to a thin layer fairly close to the galactic plane. Meanwhile, spectroscopic observations of interstellar reddening in the light of distant objects of all kinds have been made by photographic and photo-electric means. Coefficients of this selective absorption depend greatly on galactic latitude and longitude, the reddening being greatest at low latitudes and towards the galactic centre. The correlation with distance is very rough, but the value of the mean coefficient taken around the galactic circle suggests that only about one third of the total absorption is selective. Our present picture of absorbing matter in the Galaxy is one of irregular clouds close to the plane of the Milky Way superposed on a thin stratum which dims the light of stars in and behind it according to a cosecant law down to a galactic latitude of $10^{\circ}$ or so. Less than half this absorption is selective, the reddening caused by this part being due to particles scattering according to a $\lambda^{-1}$ law; the rest is non-selective blocking, presumably by larger particles. The stratum extends to at least 2,000 parsecs from the sun, but we cannot say that it necessarily permeates the Galaxy : it may be (to this extent) local. The irregular obscuring clouds closer to the plane of the Galaxy produce heavy absorption and reddening. The recognition of this absorption during the last decade has roughly halved our estimate of the size of the Galaxy and thus ineidentally brought it more nearly into accord with that of other giant systems.

Dr. H. Brïck, in a contribution on the structure of the Galaxy with special reference to the neighbourhood of the sun, took up the question of the interpretation of star-counts made up to distances of about 2,000 parsecs from the sun. The density function derived from these data, assuming the form of the luminosity function (which is fairly well known), depends greatly on the value assumed for the interstellar absorption. Away from the galactic plane the absorption is small and interpretation is not difficult, but close to the galactic equator the analysis becomes less and less easy. If the absorption is assumed negligible, the well-known Kapteyn universe results, with the sun central in a universe of stars the density of which falls to 10 per cent of its central value at 500 parsecs along the axis of symmetry of the Galaxy and at 3,000 parsecs in its plane. Charlier's "local cluster" of $B$ stars, with the sun eccentrically located 70 parsecs from the centre of a flattened system 700 parsecs in diameter and 250 parsecs thick, is now regarded as only the nucleus of a much larger cluster associated with Gould's belt. This cluster may itself be one of many star clouds which constitute the Galaxy as a whole ; certainly the observations of galactic rotation prove conclusively that the sun is located at least 8,000 parsecs from the galactic centre. The steep negative density gradients of Kapteyn's universe cannot be eliminated by the assumption of a constant coefficient of absorption : if the absorption postulated is big enough to give constant space densities out to 1,000 parsecs, then rapid rises which are quite unlikely set in farther out.

The compromise due to Bok, who uses a coefficient of 0.4 mag. $/ \mathrm{kpc}$., yields a density distribution which is roughly constant up to 1,000 parsecs in directions perpendicular to that of the galactic centre, which decreases rapidly towards the anticentre, and which first decreases (100-800 parsecs) and then rises again towards the centre. This tentative picture of a local system elongated at right angles to the direction of the galactic centre tempts us to believe that the sun is situated in a spiral arm of the Galaxy ; but Oort's work leads to a very different conclusion. This is based on observations of stars of the 10th to 17th magnitude in galactic latitudes higher than $10^{\circ}$, and is thus independent of the worst irregularities due to absorbing clouds nearer the equatorial plane. On the basic assumption that all absorption has occurred before the mean distance of the 10th magnitude stars, Oort finds that the sun is in a region of low density surrounded in all galactic longitudes by higher densities. The suggestion here is that of a 'local void' between two spiral arms rather than a local cluster, and discrimination between the two hypotheses must await further observations.

Sir Arthur Eddington then gave an account of our present knowledge of the rotation of the Milky Way. The effect of galactic rotation is observable both in the radial velocities and in the proper motions of the stars, but the data from which its amount is determined are in fact confined to the radial velocities, the proper motions providing only confirmatory evidence. In the neighbourhood of the sun, the planetary-type motion distorts a cube of stars into a rhomboid by an action analogous to a shear; differential motion is then observed between the faces of the cube, and terms of the form $A r \sin 2\left(l-l_{0}\right)$ are necessary to represent the observations. The rotational constant $A$ is about $17 \mathrm{~km}$./sec. per kiloparsec. The actual rotational velocity in the neighbourhood of the sun is obtained from radial velocities of distant clusters: it amounts to $250 \mathrm{~km}$. $/ \mathrm{sec}$., the distance to the centre of rotation being 10,000 parsecs (this agrees well with Shapley's figure when interstellar absorption is taken into account), and the total galactic mass being roughly $10^{11}$ times that of the sun. The time of one complete rotation is about $250 \times 10^{8}$ years, a figure which has remained unmodified since the pioneer work of Lindblad and Oort. 
The observations of galactic rotation throw some light on the question of the reality of the local cluster discussed by Dr. Brück. Rotational shear in such a cluster would be expected to spread it into a ringcertainly a cluster having the shape of a flattened ellipsoid of revolution would be unstable in a rotating galaxy. This objection is quite independent of theory : it is an observational fact that shear exists in the neighbourhood of the sun, hence that any local cluster must be at best a transitory phenomenon. This view is supported by observations of the cosmic cloud. The interstellar atoms and molecules responsible for stationary lines in stellar spectra are widely distributed throughout the Galaxy and show the same galactic rotation effect as the mean of the stars. It can be shown that these interstellar particles must move with velocities corresponding to circular orbits, otherwise impossibly large density gradients would be set up; hence the stars, too, are so moving, and any local cluster could not persist. From this point of view Sir Arthur welcomed Oort's hypothesis as a way of avoiding the dynamically unstable singularities involved in the hypothesis of a local cluster.

The discussion was wound up by the Astronomer Royal, Dr. H. Spencer Jones, who took as his subject the rotation of extra-galactic nebulæ and their internal distribution of mass. Pointing out that the planetary-type rotation described for our own Galaxy by Sir Arthur Eddington referred only to the solar neighbourhood, presumably at about 10,000 parsecs from the centre, Dr. Spencer Jones turned to spectroscopic observations of the rotation of other galaxies. These referred until recently only to the bright central portions of such objects as $N G C 4594$ and 4111 , the rotation of the nuclear regions of which is of the 'solid' type giving constant angular velocities.

Within the last few years, however, work at the Lick Observatory has made available rotational velocities for the Andromeda nebula ( $M$ 31) and $M 33$ out to considerable distances from their centres. The results of Babcock for $M 31$ show a period for the core of $11 \times 10^{6}$ years, and for the main body of $92 \times 10^{6}$ years, and indicate that much of the mass is in the outer regions. In $M 33$ Mayall and Aller find that the velocity increases at first linearly with radial distance from the centre, then faster until at the edge of the main body a maximum velocity is reached corresponding to a rotation period of $59 \times 10^{6}$ years. In the outer parts the velocity decreases again, the corresponding periods varying from 60 to $200 \times 10^{6}$ years. In these regions Oort's rotational "constant" $A$ varies from $84 \mathrm{~km}$. $/ \mathrm{sec}$./kpc. at 1,280 parsecs from the centre to $30 \mathrm{~km}$. $/ \mathrm{sec} . / \mathrm{kpc}$. at 1,920 parsecs, a rough extrapolation indicating that at about 2,200 parsecs conditions of rotation similar to those in the solar neighbourhood would be reached. This in turn indicates that if $M \mathbf{3 3}$ and our own Galaxy are comparable bodies, the sun is at a distance from the centre of at least twice the radius of the main body, and that from an external galaxy the main body would appear only about 8,000 parsecs in diameter, the sun being well beyond this in the thin outer regions. The observed distribution of rotational velocity in both $M 31$ and $M 33$ can be adequately represented by a thin-disk model, possessing circular symmetry, the individual parts of which revolve in circular orbits about the centre in the plane of the disk. Assuming that in such a disk the surface density can be expressed as a fifth-degree polynomial in the distance from the centre, Wyse and Mayall have determined the curves of force and thence of rota- tional velocity for typical disks. Then by working backwards they deduce the density distribution in $M 31$ and $M 33$ necessary to fit the observed velocity curves.

A wide distribution of mass throughout both systems is indicated, little tendency being found towards the central condensation usually postulated for our own Galaxy. In $M 31$, for example, the model of best fit consists of two concentric thin disks, and indicates that less than 1 per cent of the mass is contained in the central regions. The total mass is $9.5 \times 10^{10}$ suns, which agrees well with that of the Galaxy. A rediscussion of Oort's constants of galactic rotation shows that the information they yield concerning the distribution of matter in the Galaxy, while consistent with strong central condensation, does not lead uniquely to such condensation. It may well be, then, that the mass of the Milky Way system resides largely in its outer regions, in which the sun lies. The question then arises, Why should the brightness of a galactic system be so much less in the outer regions than near its nucleus? Can it be that the luminous giant stars occur near the centre while dwarfs tend to preponderate in the outer regions ; or is non-luminous dust so widespread round the periphery that it contributes much of the total mass of the Galaxy? These and many other questions of galactic structure must await further observations in happier times.

\section{BRITISH ASSOCIATION CONFERENCE ON MINERAL RESOURCES}

$\mathrm{T}$ HE Conference on Mineral Resources and the Atlantic Charter, held at the London School of Hygiene and Tropical Medicine on July 24-25, was convened by the British Association Division for the Social and International Relations of Science and, like the recent conference on agriculture, owed its origin to suggestions made at the Conference on Science and World Order held last September. The fourth clause of the Atlantic Charter promises equal access on the part of all peoples to the natural resources of the world, and as minerals are among the most important of those resources, it was felt that a survey of the situation might clarify ideas; the general state of information as to the distribution and nature of mineral deposits among the public and even, it is to be feared, in higher quarters, being very imperfect. Coal and oil were excluded from the scope of the discussion as requiring separate treatment. Those who have had occasion to examine questions concerning mineral deposits have been impressed by the unsatisfactory condition of the statistical knowledge about them which is available, in spite of excellent isolated efforts, and for that reason prominence was given to this aspect in the resolution which was put by Sir Stafford Cripps and passed at the end of the Conference. After a preamble referring to the Atlantic Charter, this ran :

"This Conference, having specifically dealt with mineral resources, submits that, as a first step, the Council should initiate forthwith consultations with appropriate scientific and technical organizations, to secure an understanding on the principles involved. The Conference would further urge that a scientific 